Editorial

\section{Uterine Fibroid Embolization in time of Covid-19}

\author{
Nestor Kisilevzky ${ }^{1 *}$ and John C Lipman² \\ ${ }^{1}$ Medical Director, Endovascular Center, São Paulo, Brazil \\ ${ }^{2}$ Medical Director, Atlanta Fibroid Center, Atlanta, USA
}

The coronavirus pandemic has caused major changes in society around the world, especially in healthcare systems. Patients with various medical ailments and conditions who were scheduled to undergo elective treatments before the pandemic arrived, wonder now if they still should follow through with it. First and foremost, if a procedure can be delayed without resulting in significant additional morbidity to the patient, it should be. The current efforts of social distancing, isolation, frequent handwashing, etc. are important steps in the fight to curtail and ultimately stop this virus. There is also a legitimate concern that performing elective procedures in time of a pandemic could increase the risk of infection to patients and medical staff, lead to shortage of personal protective equipment (PPE), or occupy a muchneeded ICU bed in the case of a significant complication. The initial result of this was the cancellation of all elective hospital surgeries and procedures. While patients with uterine fibroids are typically not threatened like the Critical Limb Ischemia patients for instance, nonetheless, some of these patient's quality of life have been significantly impacted.

Thus, the limitations imposed to access elective treatments, either to reduce cross-transmission of the virus or to prepare the hospital structure to assist infected patients, have forced women to live with discomfort and/or temporary treatments with little effectiveness. For this reason, we have continued to offer Uterine Fibroid Embolization (UFE) during the Covid-19 pandemic. As a minimally invasive procedure, UFE is commonly performed with local anesthesia and intravenous sedation in an outpatient or office-based lab, or at most an overnight hospital stay [1]. Treating these symptomatic fibroid patients with UFE vs. surgery offers a number of advantages. These include a much lower complication rate, sparing much needed ICU space, freeing up operating rooms for other procedures that must be performed in an inpatient setting, and a much lower incidence of hospital readmission. UFE is indicated as a nonsurgical treatment for symptomatic fibroids. Although fibroids can have an impact on quality of life, they are rarely life-threatening, and therefore UFE is considered an "elective" procedure. However, some special situations should
More Information

*Address for Correspondence: Nestor Kisilevzky, MD, Endovascular Center Rua Guararapes, 682, São Paulo 05077051, Brazil, Email: dr.nestor@endovascularcenter.com.br; kisilevn@uol.com.br

Submitted: 23 June 2020

Approved: 01 July 2020

Published: 02 July 2020

How to cite this article: Kisilevzky N, Lipman JC. Uterine Fibroid Embolization in time of Covid-19. Clin J Obstet Gynecol. 2020; 3: 079080 .

DOI: 10.29328/journal.cjog.1001053

Copyright: @ 2020 Kisilevzky N, et al. This is an open access article distributed under the Creative Commons Attribution License, which permits unrestricted use, distribution, and reproduction in any medium, provided the original work is properly cited.

\section{(2) Check for updates}

OPEN ACCESS

be highlighted. Women with large intramural or submucosal fibroids, tend to experience extremely heavy bleeding often accompanied by anemia that can require iron replacement or supplementation. It is not uncommon for these patients to need not only iron, but blood transfusion as well. This can be aggravated in patients with some type of natural or acquired clotting disorder or in those who, by medical recommendation, need to be anticoagulated. In these circumstances, UFE can be considered a "non-elective" procedure. While this hemorrhagic condition is not life-threatening to most patients, it may force them to go to the Emergency Room; risking Covid-19 exposure, as well as unnecessary additional testing, and perhaps even an emergency hysterectomy. The iron deficiency anemia that fibroid patients typically suffer with is gradual, but progressive. The symptoms of fatigue and lethargy may even be falsely attributed by the patient to age or because she has suffered through this for so long, it becomes her "new normal." These facts should not minimize the significance of the anemia, which if profound, is associated with a higher risk of acute myocardial infarction, stroke, and cerebrovascular disease [2]. Therefore, improving anemia should mitigate these risks. While correction of the anemia is helpful, fixing the root cause of the anemia with UFE also appears to be justified here as well. However, people are understandably concerned about going to the hospital to receive medical care and being exposed to Covid-19. In an April study of the Journal of the American College of Cardiology, ST-segment elevation myocardial infarction (STEMI) rates dropped 38\% 
after March $1^{\text {st }}$ after the pandemic hit in United States [3]. A number of news reports point to coronavirus worries as the reason for this hospital avoidance. Many hospital admissions are down 25\% - 40\%. Hospitals have responded to these concerns by establishing strict traffic flows and increased sanitation protocols.

However, an alternative to avoiding hospitals altogether are outpatient clinics or office-based centers where UFE can be performed safely and more efficiently. There is not only much lighter traffic, they do not care for or house Covid-19 patients. Therefore, this should be a much safer environment for patients that can be safely treated in the outpatient setting. Safety of the staff and patients begins with the supply of proper PPE, availability of adequate testing, and developing explicit protocols on how to minimize the risk of potential infection. It is worth mentioning that UFE is performed under conscious sedation through femoral or radial access. There is no need for tracheal intubation or laryngeal mask or any other procedure that may cause spread of respiratory droplets that is well known to be the source of contamination by Covid-19.

Uterine fibroids are benign tumors which typically exhibit slow, continual growth. But some patients exhibit more rapid growth which is not well understood, and in the past was worrisome for an occult malignancy. While no one knows where fibroids originate, their growth is tied primarily to estrogen. Therefore, hyperestrogenic states like obesity or exogenous estrogen through medication or even the environment can cause these benign tumors to grow more rapidly and may accelerate the patient's symptoms. For these patients, delaying the procedure may be unwarranted.

While the vastmajority of UFE patients recover uneventfully over 5-7 days, there are a small percentage that will suffer with a postembolization syndrome characterized by pelvic pain, low grade fever, and nausea. This can result in readmission to the hospital. Many analgesia protocols to reduce postoperative pain have been described in the literature which include the use of opioids, non-steroidal anti-inflammatories drugs, intra-arterial lidocaine injection, superior hypogastric nerve block among others [4]. These protocols to address post-embolization pain in advance has considerably reduced the need for hospital readmission. However, to improve postprocedure communication between patients and medical staff, new channels through teleconferencing tools have been established, which allows patients to continue to be assisted remotely avoiding unnecessary trips to the medical office or hospital.

In summary, women who suffer from symptoms of uterine fibroids, especially those with significant bleeding and anemia, can undergo treatment with UFE despite the Covid-19 pandemic. The alternative of performing UFE in outpatient clinics or office-based labs may offer a sure option to reduce the risk of infection with Covid-19 and it may ultimately be where all UFEs are done in the future.

\section{References}

1. Keung JJ, Spies JB, Caridi TM. Uterine artery embolization: A review of current concepts. Best Pract Res Clin Obstet Gynaecol. 2018; 46: 66-73. PubMed: https://pubmed.ncbi.nlm.nih.gov/29128204/

2. Lee G, Choi S, Kim K, et al. Association Between Changes in Hemoglobin Concentration and Cardiovascular Risks and All-Cause Mortality Among Young Women. J Am Heart Assoc. 2018; 21; 7: e008147. PubMed: https://www.ncbi.nlm.nih.gov/pubmed/30369312

3. Rodriguez-Leor O, Cid-Alvarez B. STEMI care during COVID-19: losing sight of the forest for the trees. JACC Case Reports 2020 (in press). PubMed: https://www.ncbi.nlm.nih.gov/pmc/articles/PMC7177148/

4. Saibudeen A, Makris GC, Elzein A, Wigham A, Patel R, et al. Pain Management Protocols During Uterine Fibroid Embolisation: A Systematic Review of the Evidence. Cardiovasc Intervent Radiol. 2019; 42: 1663-1677.

PubMed: https://pubmed.ncbi.nlm.nih.gov/31520113/ 